\title{
FINITE- $N$ DYNAMICS ADMIT NO TRAVELING-WAVE SOLUTIONS FOR THE HAMILTONIAN XY MODEL AND SINGLE-WAVE COLLISIONLESS PLASMA MODEL
}

\author{
YVES ELSKENS ${ }^{1}$
}

\begin{abstract}
For the hamiltonian dynamical XY model and for the self-consistent wave-particle model, the finite- $N$ analogues of Bernstein-Greene-Kruskal solutions of the Vlasov equation would be travelingwave solutions with nontrivial motion of the particles. Such solutions do not exist because they would exhibit singularities in complex time which are not compatible with the traveling-wave assumption.

Résumé. Pour le modèle hamiltonien XY dynamique et pour le modèle auto-cohérent onde-particules, les analogues pour $N$ fini des solutions de Bernstein-Greene-Kruskal de l'équation de Vlasov seraient des solutions en onde progressive avec un mouvement non trivial des particules. Ces solutions n'existent pas, car elles présenteraient des singularités en temps complexe incompatibles avec l'hypothèse de l'onde progressive.
\end{abstract}

AMS Subject Classification. 70F 10, 82C05, 82C40.

\section{Motivation}

The kinetic description of plasmas by the Vlasov equation provides much insight to their dynamics in the limit of large number of interacting particles. In this study, Bernstein-Greene-Kruskal (BGK) solutions [2] play an important role as the prototype of exact solutions of the self-consistent nonlinear Vlasov-Poisson system. Their discovery was a breakthrough in the study of this system, and they also appear today as asymptotic regimes for time-dependent solutions.

On the other hand, as actual statistical mechanics systems are made of finite (yet large !) numbers of particles, it may be desirable to understand how robust the predictions of kinetic theory are to the passage to finite $N$ models. In this paper we discuss two simple models : the dynamical XY model, and the self-consistent $M=1$ wave-particle model.

Whereas the kinetic limits of these models admit BGK solutions, we show that, $\forall N<\infty$, these models admit no solutions with a time-independent nonvanishing field and nontrivial particle motion. It should be noted that both models belong to the mean-field class, so that the formal limit $N \rightarrow \infty$ commutes with time evolution (see [11] for the XY model and [6] for the wave-particle model).

\footnotetext{
Keywords and phrases: Hamiltonian dynamics, Kinetic equations, Exact solutions, Collisionless plasmas

${ }^{1}$ Physique des interactions ioniques et moléculaires, Unité 6633 CNRS-Université de Provence,

Equipe turbulence plasma, case 321, campus de Saint-Jérôme, F-13397 Marseille cedex 13

e-mail: elskens@newsup.univ-mrs.fr
}

(c) EDP Sciences, SMAI 2001 


\section{Models And Results}

The dynamical XY model [10] is described by the $N$-degrees-of-freedom hamiltonian

$$
H_{\mathrm{XY}}=\sum_{r=1}^{N} \frac{p_{r}^{2}}{2}+\frac{\kappa}{2 N} \sum_{r=1}^{N} \sum_{j=1}^{N}\left(1-\cos \left(x_{r}-x_{j}\right)\right)
$$

with conjugate variables $\left(x_{r}, p_{r}\right)$. The single-wave model for self-consistent wave-particle interaction $[4,3]$ has the hamiltonian with $N+1$ degrees of freedom

$$
H_{\mathrm{sc}}=\sum_{r=1}^{N} \frac{p_{r}^{2}}{2}+\omega_{0} I-\frac{\beta}{\sqrt{N}} \sum_{r=1}^{N} \sqrt{2 I} \cos \left(x_{r}-\theta\right)
$$

with conjugate variables $\left(x_{r}, p_{r}\right)$ for particles and $(\theta, I)$ for the wave. The coupling constants are $\kappa \neq 0$ and $\beta>0$ respectively. The positions $x_{r}$ and the phase $\theta$ are on the circle $S=\mathbb{R} /(2 \pi \mathbb{Z})$. The momentum space is $\mathbb{R}$ for the particles, and the wave intensity $I \in\left[0,+\infty\left[.^{1}\right.\right.$

An important quantity for both models is the total field

$$
E=N^{-1} \sum_{r=1}^{N} \mathrm{e}^{\mathrm{i} x_{r}}
$$

These hamiltonians generate the particle equations of motion

$$
\begin{aligned}
& \dot{x}_{r}=p_{r} \\
& \dot{p}_{r}=-A \sin \left(x_{r}-\theta\right)
\end{aligned}
$$

where for the XY model

$$
A \mathrm{e}^{\mathrm{i} \theta}=\kappa E
$$

For the wave-particle system, the equations of motion read the same, but

$$
A \mathrm{e}^{\mathrm{i} \theta}=\beta Z^{*}
$$

where we introduce the complex variable for the wave

$$
Z=\sqrt{\frac{2 I}{N}} \mathrm{e}^{-\mathrm{i} \theta}
$$

Here the wave is a dynamical degree of freedom with its own evolution equation, which we write (the star denoting the complex conjugate)

$$
\dot{Z}=-\mathrm{i} \omega_{0} Z+\mathrm{i} \beta E^{*}
$$

We seek solutions of (4)-(5) with either (6) or (7)-(9), in which the force on a particle would be the same as if it were due to a traveling wave, i.e. in which $A$ and $\dot{\theta}$ would be constant. Then a Galileo transformation would allow setting $\dot{\theta}=0$ in the appropriate frame, and $\theta$ would also be constant.

\footnotetext{
${ }^{1}$ One can avoid the singularity with respect to $I$ using cartesian conjugate variables $(X, Y)$ for the wave, so that $Z=X+\mathrm{i} Y$. This is less convenient to our purpose.
} 
For $N \geq 2$, exact solutions exist with $A=0$ and, for (2), $Z=0$. The particles move at constant velocities in beams of equally spaced particles. More precisely, write $N$ as a sum of $b$ integers $N_{s} \geq 2$, associate to each beam $s$ a velocity $v_{s}$ and positions $x_{r}=\theta_{s}+v_{s} t+2 \pi r / N_{s}$ : this ensures that $E=0$ for all times. This multibeam family of exact solutions has been useful in discussing

- for the XY model [1], the reduction of the many-body dynamics to effective wave-particle self-consistent dynamics with fewer degrees of freedom,

- for the wave-particle model [4], the difference between Landau damping and growth from the viewpoint of hamiltonian dynamics ${ }^{2}$.

Theorem 1.1. For any $N<\infty$, the only solutions to the system (4)-(5)-(6) or to the system (4)-(5)-(7)-(9), such that $A>0$ and $\theta$ constant, are the trivial solutions $x_{r} \in\{\theta, \theta+\pi\}, p_{r}=0 \forall 1 \leq r \leq N$.

Remark 1.2. We call these solutions trivial because they are equivalent to considering at most two macroparticles with mass $N_{1}$ at $\theta$ and $N_{2}=N-N_{1}$ at $\theta+\pi$. This is no analogue to a kinetic-theoretical BernsteinGreene-Kruskal mode with a well behaved density in $(x, p)$ space.

\section{TRivial SOlutions}

For the trivial solutions, $E=\left(2 N_{1} / N-1\right) \mathrm{e}^{\mathrm{i} \theta}$. The case $N=2 N_{1}$ is a special case of the multibeam solution. For the both models, $x_{r}=\theta$ or $x_{r}=\theta+\pi$ implies that $\dot{p}_{r}=0$.

For the XY model, condition $p_{r}=0$ then ensures that $(A, \theta)$ remains constant.

For the wave-particle model, the constant $(A, \theta)$ condition reads $\dot{Z}=0$, which reduces to $\omega_{0} Z=\beta E^{*}$.

\section{Nonexistence of OTHER SOlutions}

Proof. Our proof is by contradiction. Consider a nontrivial solution and let $\theta=0$ with no loss of generality. We shall show that $E$ should satisfy contradictory requirements, being a constant function of time and exhibiting infinitely many singularities in complex time.

For the wave-particle model (2), the constant $(A, \theta)$ condition reads $\omega_{0} Z=\beta E^{*}$, so that $E$ must be constant. For the XY model (1), the constant $(A, \theta)$ condition directly implies constant $E$.

For constant $(A, \theta)$, each particle follows a solution of the pendulum equation, i.e. one of the following four functions of time :

1. $x_{r}=0$ or $x_{r}=\pi$;

2. $x_{r}=2 \operatorname{Arcsin}\left[k_{r} \operatorname{sn}\left(\sqrt{A}\left(t-t_{r}\right), k_{r}\right)\right]$ with $0<k_{r}<1$ and $t_{r} \in \mathbb{R}$;

3. $x_{r}=4 \operatorname{Arctg} \mathrm{e}^{\sigma_{r} \sqrt{A}\left(t-t_{r}\right)}$ with $\sigma_{r}= \pm 1$ and $t_{r} \in \mathbb{R}$;

4. $x_{r}=2 \operatorname{am}\left(\sigma_{r} k_{r}^{-1} \sqrt{A}\left(t-t_{r}\right), k_{r}\right)$ with $\sigma_{r}= \pm 1,0<k_{r}<1$ and $t_{r} \in \mathbb{R}$.

If only case 1 were realized, we would have a trivial solution. Thus we now prove that no particle moving according to cases 2, 3 and 4 is allowed. We first note that all these functions are analytic with respect to complex time in a neighbourhood of the real axis. As (3) is analytic with respect to positions $x_{r}, E$ is analytic with respect to $t$ in this neighbourhood.

Consider first case 4 (circulating particles). The am function has logarithmic singularities (thus $x_{r}$ is not meromorphic), but

$$
\mathrm{e}^{\mathrm{i} x_{r}}=2 \mathrm{cn}^{2} u_{r}-1+2 \mathrm{i} \operatorname{sn} u_{r} \operatorname{cn} u_{r}
$$

with $u_{r}=\sigma_{r} A^{1 / 2}\left(t-t_{r}\right) / k_{r}$, so that $\mathrm{e}^{\mathrm{i} x_{r}}$ is meromorphic. Thus $\mathrm{e}^{\mathrm{i} x_{r}}$ is periodic in $t$ (with periods $2 \mathrm{~K}\left(k_{r}\right)$ and $4 \mathrm{iK}^{\prime}\left(k_{r}\right)$ in $\left.u_{r}\right)$, and it has double poles at $u_{r}= \pm \mathrm{iK}^{\prime}\left(k_{r}\right)$. The location of the poles in complex $t$ plane determines

\footnotetext{
${ }^{2}$ In kinetic theory, Landau damping and Landau growth are described using the linearized Vlasov equation, for the small perturbations to a vanishing field solution $f(x, p, t)=F(p)$. But with a finite number of degrees of freedom, no solution to hamiltonian dynamics can admit exponentially damped perturbations without the existence of exponentially unstable perturbations.
} 
uniquely $k_{r}$ (from the period $2 A^{-1 / 2} k_{r} \mathrm{~K}\left(k_{r}\right)$ ) and $t_{r}$ (up to a period) ; the direction of the motion determines $\sigma_{r}=\operatorname{sign} p_{r}$. Thus there is a one-to-one mapping between the singularities of $\mathrm{e}^{\mathrm{i} x_{r}}$ and the parameters of the motion. Therefore no distribution of other particles can produce a sum $\sum_{j} \mathrm{e}^{\mathrm{i} x_{j}}$ able to cancel the singularities associated with a given particle.

Case 2 (librating particles) reduces to case 4 if one sets $k_{r}>1$ in case 4 . The previous discussion applies.

Finally, for case 3 (homoclinic particles),

$$
\mathrm{e}^{\mathrm{i} x_{r}}=\frac{\left(1+\mathrm{i} w_{r}\right)^{2}}{\left(1-\mathrm{i} w_{r}\right)^{2}}
$$

with $w_{r}=\mathrm{e}^{u_{r}}$ and $u_{r}=\sigma_{r} A^{1 / 2}\left(t-t_{r}\right)$. Then $\mathrm{e}^{\mathrm{i} x_{r}}$ is meromorphic, has a double pole at $u_{r}=-\mathrm{i} \pi / 2$ and is simply periodic in $u_{r}$ with period $2 \pi \mathrm{i}$. Again, there is a one-to-one correspondence between each particle and its family of singularities (which all have the same period $2 \mathrm{i} \pi A^{-1 / 2}$ and differ by $\sigma_{r}=\operatorname{sign} p_{r}$ and $t_{r}$ ).

To conclude, note that for a finite number of homoclinic particles, their poles form a finite family of simplyperiodic arrays of poles, whereas any circulating or librating particle is associated with a doubly infinite array of poles. Thus, for a finite total number $N$ of particles, no compensation can occur between homoclinic particles and other particles.

This completes the proof.

Remark 3.1. Consider $N_{\text {hom }}$ homoclinic particles. They all asymptote the fixed saddle point of the pendulum potential, so that $x_{r}= \pm \pi y_{r} \mathrm{e}^{-\sqrt{A} t}+\mathcal{O}\left(\mathrm{e}^{-2 \sqrt{A} t}\right)$ as $t \rightarrow \infty$, for some finite $y_{r}$. Then they contribute to $E$ with

$$
E_{\text {hom }}=N^{-1} \sum_{r} \mathrm{e}^{\mathrm{i} x_{r}}=N^{-1}\left(N_{\text {hom }}+\sum_{r} \mathrm{i} y_{r} \mathrm{e}^{-\sqrt{A} t}+\mathcal{O}\left(\mathrm{e}^{-2 \sqrt{A} t}\right)\right)
$$

which clearly is a nonconstant function of time.

\section{BGK solutions FOR The Vlasov EQUATION IN THE Kinetic Limit}

Let us finally recall explicit traveling solutions of the kinetic equations which describe our systems in the limit $N \rightarrow \infty$. This kinetic limit is described by a distribution function $f(x, p, t)$ satisfying the Vlasov equation

$$
\partial_{t} f+p \partial_{x} f-A \sin (x-\theta) \partial_{p} f=0
$$

The total field is $E(t)=\int_{S \times \mathbb{R}} f(x, p, t) \mathrm{d} p \mathrm{~d} x$. Then (6) holds for the XY model. For the wave-particle model, (7), (8) and (9) hold too (and one should scale $I=N \psi$ with $\psi=\mathcal{O}(1)$ in the limit $N \rightarrow \infty$ ).

A canonical (not the general) Bernstein-Greene-Kruskal solution traveling at velocity $v$ is found with a factorized distribution

$$
f(x, p, t \mid v, T)=\frac{1}{C(T)} \exp \left(-\frac{(p-v)^{2}}{2 T}-\frac{A}{T}(1-\cos (x-v t))\right)
$$

with $C(T)=(2 \pi)^{3 / 2} T^{1 / 2} \mathrm{e}^{-A / T} \mathrm{I}_{0}\left(\frac{A}{T}\right)$ normalizing $f$ to unity. The parameters are the velocity $v \in \mathbb{R}$ and the temperature $T>0$. For $A=0$, this is just the Maxwell velocity distribution with uniform spatial distribution.

Then the field reads

$$
E=\frac{\mathrm{I}_{1}(A / T)}{\mathrm{I}_{0}(A / T)} \mathrm{e}^{\mathrm{i} v t}
$$

For the XY model $[9,10],(6)$ can be satisfied with $A>0$ iff $0<2 T<\kappa$. Then $\theta=v t$.

For the wave-particle model [5, 7], the traveling solution requires $\theta=v t, v<\omega_{0}$ and $0<2 T<\beta^{2} /\left(\omega_{0}-v\right)$. This requirement admits a physical interpretation in the dynamics of the wave-particle system [8]. 
This analysis was motivated by discussions with F. Doveil and D.F. Escande. Discussions with C. Silvy, with MC. Firpo and with the participants to CEMRACS'99 are gratefully acknowledged. It is a pleasure to thank F. Coquel and S. Cordier for organizing the workshop at CIRM-Luminy.

\section{REFERENCES}

[1] Antoni M, Elskens Y and Escande D F 1998 Explicit reduction of N-body dynamics to self-consistent particle-wave interaction, Phys. Plasmas 5 841-852

[2] Bernstein I B, Greene J M and Kruskal M D 1957 Exact nonlinear plasma oscillations, Phys. Rev. 108 546-550

[3] Elskens Y, Guyomarc'h D and Firpo M-C 1998 Phase space dynamics and wave-particle interaction, Physicalia Mag. 20 193-203

[4] Escande D F, Zekri S and Elskens Y 1996 Intuitive and rigorous microscopic description of spontaneous emission and Landau damping of Langmuir waves through classical mechanics, Phys. Plasmas 3 3534-3539

[5] Firpo M-C 1999 Etude dynamique et statistique de l'interaction onde-particule, Thèse de doctorat, université de Provence (Marseille)

[6] Firpo M-C and Elskens Y 1998 Kinetic limit of $N$-body description of wave-particle self-consistent interaction, J. Stat. Phys. 93 193-209

[7] Firpo M-C and Elskens Y 2000 Phase transition in the collisionless damping regime for wave-particle interaction, Phys. Rev. Lett. 84 3318-3321

[8] Firpo M-C and Elskens Y 2001 O'Neil's threshold for collisionless nonlinear Landau damping and phase transition in the wave-particle system, ESAIM Proc. 11 217-222 http://www.emath.fr/Maths/Proc/Vol.10

[9] Inagaki S 1993 Thermodynamic stability of modified Konishi-Kaneko system, Prog. Theor. Phys. 90 577-584

[10] Latora V, Rapisarda A and Ruffo S 1999 Chaos and statistical mechanics in the hamiltonian mean-field model, Physica D $13138-54$

[11] Spohn H 1991 Large scale dynamics of interacting particles (Springer, New York) 